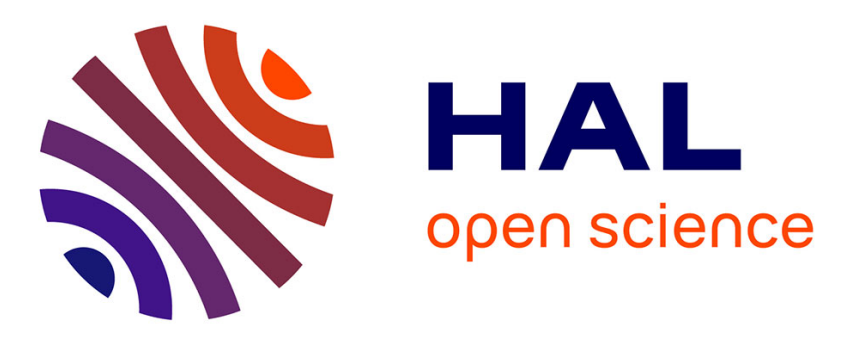

\title{
Estimating Fiedler value on large networks based on random walk observations
}

\author{
Alexandre Reiffers-Masson, Thierry Chonavel, Yezekael Hayel
}

\section{To cite this version:}

Alexandre Reiffers-Masson, Thierry Chonavel, Yezekael Hayel. Estimating Fiedler value on large networks based on random walk observations. ICASSP 2021: IEEE International Conference on Acoustics, Speech and Signal Processing, Jun 2021, Toronto, Canada. 10.1109/ICASSP39728.2021.9413713 . hal-02974433

\section{HAL Id: hal-02974433 \\ https://imt-atlantique.hal.science/hal-02974433}

Submitted on 21 Oct 2020

HAL is a multi-disciplinary open access archive for the deposit and dissemination of scientific research documents, whether they are published or not. The documents may come from teaching and research institutions in France or abroad, or from public or private research centers.
L'archive ouverte pluridisciplinaire HAL, est destinée au dépôt et à la diffusion de documents scientifiques de niveau recherche, publiés ou non, émanant des établissements d'enseignement et de recherche français ou étrangers, des laboratoires publics ou privés. 


\title{
Estimating Fiedler value on large networks based on random walk observations
}

\author{
Alexandre Reiffers-Masson, Thierry Chonavel \\ Lab-STICC, UMR CNRS 6285, Brest, France, \\ IMT Atlantique, Plouzané, France
}

\author{
Yezekael Hayel \\ LIA, \\ University of Avignon, Avignon, France
}

\begin{abstract}
In this paper, we describe an iterative scheme which is able to estimate the Fiedler value of a network when the topology is initially unknown. The only available information is the one obtained through a random walk process over the network. Our algorithm is based on the Rayleigh quotient optimization problem and the theory of stochastic approximation. We explain the different tools used to construct our algorithm and we describe our iterative scheme. Finally, we illustrate its performance through a numerical study.
\end{abstract}

Index Terms

Fiedler value, stochastic approximation, random walk based observations.

\section{INTRODUCTION}

Understanding the connectivity of large scale networks is essential for the study of their performance. Especially, in the context of overlay networks [1], [2], ad-hoc wireless networks [3] and consensus/swarming algorithms for distributed networks [4], such understanding is critical. In overlay networks, connectivity between the nodes is crucial for a better load balancing and in general for a better quality of service. A classical example of such networks are P2P networks used for file sharing and content distribution application [1], [2]. In distributed systems (distributed ledgers for instance) it is well known that the connectivity of the network is crucial to speed-up the convergence of distributed algorithms running over the network (see [4] for an application to consensus algorithms). Spectral graph theory [5] is a classical approach to study the connectivity of a network using graph analysis. The eigenvalue decomposition of a Laplacian matrix provides powerful heuristics for solving the RatioCut problem [6] and therefore can be also used to solve clustering problem in networks [6], for critical links identifications [1], [7] and to design strongly connected networks [8].

Set-up: One of the main quantities of interested in such problems is the Fiedler value [9] (and the Fielder vector associated to it). The Fiedler value is defined as the non-trivial second smallest eigenvalue of the Laplacian matrix and the Fiedler vector is the associated eigenvector. In this paper, we answer the following question about this connectivity metric: Is it possible to learn efficiently the Fiedler value and the Fielder vector of a large network by only using the information gathered by a random walk evolving on the graph? In our setup, we assume that it is not possible to have an initial full observation of the global network, and the only available information is the one obtained through a random walk process over the graph. Random walk is a standard scheme used to sample nodes and edges in a network (see [10] and the references therein). Moreover, we would like to derive efficient algorithms, which are able to manage efficiently the memory/storage of the devices used to run our algorithms.

Contributions: The main contribution of the paper is the design of an efficient iterative scheme which converges to the Fiedler vector. The information used to learn the Fiedler vector is obtained using a random walk, which discovers, step-by-step the topology of the network. The convergence of the algorithm can be deduced from recent results in stochastic approximation [11]. To be able to use the theory of stochastic approximation we show the equivalence between our problem and the problem of computing the leading eigenvector of a particular subset of matrices.

Related works: Our problem belongs to the class of works related to online/streaming computation of eigenvectors in a large-scale setting. First, solving large-scale eigenvalue problems using deterministic methods is a well studied problem, and a summary of the different methods can be found in [12]. In these lecture notes, the streaming context with stochastic feedbacks is not discussed. Then the different works on the online leading eigenvector problem [13] and online/streaming principal component analysis [14], [15] are also close to our set-up. These algorithms are more oriented towards Principal Component Analysis than the computation of Fiedler vectors. Also, our problem can find some similarities with the online distributed computation of leading eigenvectors [3], [16], [17]. In these papers, the authors propose distributed algorithms to track the Fiedler value of a stochastic network based on an extension of the power iteration algorithm. A recent survey of these different methods can be found in [17]. Another approach, similar to ours, is the one proposed in [18], [19] based on stochastic approximation theory to compute particular eigenvectors. In [18], the authors derive a stochastic approximation variant of the gossip algorithm, applied to the computation of the leading eigenvector of a nonnegative matrix. Along the same lines, 
the authors of [19] proposed a stochastic approximation algorithms inspired from Oja's algorithm. Different extensions are suggested, especially related to Spectral graph clustering. The main difference between our work and the papers cited above is the fact that we observe the network through a random walk exploration process. This kind of observations cannot be handled in the same way as the ones described in the previous works, therefore, forced us to come up with a different approach.

Finally, to the best of our knowledge, we have only identified two papers [20], [21] that use random walks to compute the Fiedler vector. In [20], a stochastic process that converges to the Fiedler vector is suggested. Its construction is based on a set of interacting random walks. We also mention that this paper contains, from the best of our knowledge, an up to date survey of the different algorithms that compute the Fiedler value and the Fiedler vector. Their approach is different than ours, in their case they use $2 n$ competitive random walks, where $n$ is the number of nodes. In our case we only need information gathered by one random walk. In [21], an algorithm is derived from a random walk and the authors proved that the algorithm converges to a local approximation of the Fiedler vector. In our case, we converge exactly to the Fiedler vector.

\section{PRoblem Definition}

In this paper, we are interested in the following question: Is it possible, using only random walk based observations of a graph topology, to estimate the Fiedler value and the associated eigenvectors of an undirected graph? In this section, we will first provide basic definitions and then we will describe our observation model. Finally, we will formulate the estimation problem that we want to solve.

Let $G:=(V, E)$ be an undirected unweighted graph, where $V:=\{1, \ldots, n\}$ is the set of nodes and $E$ is the set of edges. The adjacency matrix of $G$ is the matrix $\boldsymbol{A}:=\left[a_{i j}\right]_{i, j=1: n}$, where the $i j$-entry of $\boldsymbol{A}$ is given by $a_{i j}:=1_{(i, j) \in E}$. The neighborhood of a node $i$ is the set $\mathcal{N}(i):=\left\{j \in V \mid a_{i j}=1\right\}$. We assume that for all node $i, a_{i i}=0$. The degree of a node $i$ is given by $\operatorname{deg}(i):=\sum_{i^{\prime}=1}^{n} a_{i i^{\prime}}$ and the degree matrix is equal to $\boldsymbol{D}:=\left[\delta_{i j} \operatorname{deg}(i)\right]_{i, j=1: n}$ where $\delta$.. is the Kronecker delta operator. We also define the Laplacian matrix as $\boldsymbol{L}:=\boldsymbol{D}-\boldsymbol{A}$.

The spectrum of the Laplacian matrix will be used to measure the connectivity of the graph. More precisely, we are interested in the estimation of the second smallest eigenvalue of $\boldsymbol{L}$ denoted by $\lambda^{*}$. We assume that the graph is connected and therefore $\lambda^{*}$ is strictly positive [22]. $\lambda^{*}$ is called the algebraic connectivity (or Fiedler value) and we denote by $v^{*}$ the associated eigenvector. In this work, a discrete time random walk $X(k)$ is used to explore the graph step-by-step. At each time step $k$, the stochastic process $X$ is in vertex $i_{k} \in V$ and the neighboring nodes $\mathcal{N}\left(i_{k}\right)$ are observable. Then, after the $k$-th step of the random walk process, the $\sigma$-algebra $\mathcal{H}(k)$ is given by $\mathcal{H}(k):=\left\{i_{1}, \mathcal{N}\left(i_{1}\right), \ldots, i_{k}, \mathcal{N}\left(i_{k}\right)\right\}$ where for all $k \geq 0$ the node $i_{k}$ is sampled following the law $\boldsymbol{\pi}_{0} \boldsymbol{P}^{k}$ with $\boldsymbol{P}=\boldsymbol{D}^{-1} \boldsymbol{A}$ and with $\boldsymbol{\pi}_{0}$ being the initial distribution of the random walk process. The goal of this work is to derive, for every $k \geq 0$, a consistent estimator of $\lambda^{*}$ (resp. $\left.v^{*}\right)$ denoted by $\lambda(\mathcal{H}(k))($ resp. $v(\mathcal{H}(k))$ ).

\section{COMPUTATION OF THE FIELDER VALUE AS A LEADING EIGENVECTOR PROBLEM}

To be able to derive an efficient estimator of the Fiedler value and the Fiedler vector, we consider a dynamical system approach. We first recall that the leading eigenvalue and the associated eigenvector is the solution of an optimization problem over the unit sphere. Then we describe the ordinary differential equations which converges to the solution of this optimization problem. We will next show that the computation of the Fiedler vector can be reformulated as a leading eigenvector problem. Finally, we describe a stochastic approximation which is tracking the O.D.E. and therefore converges to the Fiedler vector.

\section{A. Rayleigh quotient optimization problem}

For any matrix $W$, the Rayleigh quotient $r_{W}: \mathbb{R}^{n} \rightarrow \mathbb{R}$ is the smooth function given by:

$$
r_{\boldsymbol{W}}(\boldsymbol{x})=\boldsymbol{x}^{T} \boldsymbol{W} \boldsymbol{x}\|\boldsymbol{x}\|^{-2} .
$$

Let $U^{n-1}:=\left\{\boldsymbol{x} \in \mathbb{R}^{n} \mid\|\boldsymbol{x}\|=1\right\}$ be the unit sphere in $\mathbb{R}^{n}$. Then by considering the restriction of the Rayleigh quotient on the unit sphere $U^{n-1}$, the eigenvalues of a real symmetric matrix are characterized using the Rayleigh Quotient [23] and in particular:

$$
\lambda_{\min }(\boldsymbol{W})=\min _{\boldsymbol{x} \in U^{n-1}} r_{\boldsymbol{W}}(\boldsymbol{x}) .
$$

Therefore, the largest eigenvalue of $\boldsymbol{W}$ can be obtained by solving an optimization problem on the manifold $U^{n-1}$. One approach to solve this optimization problem is to apply the gradient flow in order to search for the dominant eigenvector of $\boldsymbol{W}$. This leads to the well known Rayleight Quotient Gradient Flow [24] defined as the following ordinary differential equations:

$$
\dot{\boldsymbol{x}}=-\left(\boldsymbol{W}-r_{\boldsymbol{W}}(\boldsymbol{x}) I_{n}\right) \boldsymbol{x}, \boldsymbol{x}(0)=\boldsymbol{x}_{0} \in U^{n-1}, .
$$

It can be proved that under specific conditions $\left(\lambda_{\min }<\lambda_{2}, \boldsymbol{W}\right.$ is a symmetric matrix) that $\boldsymbol{x}$ converges to the eigenvector associated to the eigenvalue $\lambda_{\min }$ (see [24]). 


\section{B. Problem transformation}

In this section we explain how to transform the problem of tracking the second eigenvector (in increasing order of the eigenvalues) of the Laplacian matrix $\boldsymbol{L}$ into the one of tracking the first eigenvector of a modified positive matrix.

Let $\left\{\lambda_{i}\right\}_{i=1: n}$ represent the eigenvalues of $\boldsymbol{L}$, with $\lambda_{1}<\lambda_{2}<\lambda_{3} \leq \ldots \leq \lambda_{n}$, and $\boldsymbol{v}_{1}, \ldots, \boldsymbol{v}_{n}$ the corresponding unit norm eigenvectors. Recall that $\lambda_{1}=0$ and $\boldsymbol{v}_{1}=\frac{1}{\sqrt{n}} \mathbb{I}$, with $\mathbb{I}=[1, \ldots, 1]^{T}$. Then, tracking $\boldsymbol{v}_{2}$ can be done equivalently by tracking the eigenvector associated with the largest eigenvalue of the matrix $\boldsymbol{I}-\frac{1}{n} \mathbb{\Pi} \mathbb{I}^{T}-\epsilon \boldsymbol{L}$ for $\epsilon<\lambda_{n}^{-1}$. This method is called deflation and it is proposed in [3]. When $\lambda_{n}$ is large this could lead to numerical issues. Alternatively, we propose to work with the restriction of $\boldsymbol{L}$ to the space orthogonal to vector $\mathbb{I}$. Clearly a basis of this subspace is given by the columns of the matrix $\boldsymbol{M} \in \mathbb{R}^{n \times n-1}$ with $\boldsymbol{M}_{i i}=1, \boldsymbol{M}_{i+1, i}=-1$ and other entries equal to 0 .

Proposition 1. Let denote by $\lambda_{1}<\lambda_{2}<\lambda_{3} \leq \ldots \leq \lambda_{n}$ the eigenvalues of a Laplacian matrix $L$ and $\boldsymbol{v}_{1}, \ldots$, $\boldsymbol{v}_{n}$ corresponding unit norm eigenvectors. Then, we consider the following matrix:

$$
\boldsymbol{S}=\boldsymbol{Q}^{T} \boldsymbol{L} \boldsymbol{Q}
$$

with $\boldsymbol{Q}=\left[\boldsymbol{q}_{1}, \ldots, \boldsymbol{q}_{n-1}\right] \in \mathbb{R}^{n \times n-1}$ and

$$
\boldsymbol{q}_{k}=\frac{1}{\sqrt{k(k+1)}}[1, \ldots, 1,-k, 0, \ldots, 0]^{T},
$$

( $k$ entries are equal to $\sqrt{k(k+1)}^{-1}$ ). The eigenvalues of matrix $S$ are $\lambda_{2}<\lambda_{3} \leq \ldots \leq \lambda_{n}$ and corresponding unit norm eigenvectors $\boldsymbol{Q}^{T} \boldsymbol{v}_{2}, \ldots, \boldsymbol{Q}^{T} \boldsymbol{v}_{n}$.

Proof The columns of $\boldsymbol{Q}$ are unit norm vectors, orthogonal to II and to each other: $\boldsymbol{q}_{i} \perp \boldsymbol{q}_{j}$ for $i \neq j$. Let $\boldsymbol{L}=\boldsymbol{V} \boldsymbol{\Lambda} \boldsymbol{V}^{T}$ denote the EVD of $\boldsymbol{S}$, where $\boldsymbol{\Lambda}=\operatorname{diag}\left(\lambda_{1}, \lambda_{2} \ldots \lambda_{n}\right)$. Then, we have

$$
\boldsymbol{Q}^{T} \boldsymbol{V}=\left[\mathbf{0}, \boldsymbol{Q}^{T} \boldsymbol{v}_{2}, \ldots, \boldsymbol{Q}^{T} \boldsymbol{v}_{n}\right] .
$$

Since in addition the matrix $\tilde{\boldsymbol{Q}}=[\mathbb{I} \mid \boldsymbol{Q}]$ is orthogonal, for $1<i, j \leq n$ we have

$$
\left(\boldsymbol{Q}^{T} \boldsymbol{v}_{i}\right)^{T}\left(\boldsymbol{Q}^{T} \boldsymbol{v}_{j}\right)=\left(\tilde{\boldsymbol{Q}}^{T} \boldsymbol{v}_{i}\right)^{T}\left(\tilde{\boldsymbol{Q}}^{T} \boldsymbol{v}_{j}\right)=\boldsymbol{v}_{i}^{T} \tilde{\boldsymbol{Q}} \tilde{\boldsymbol{Q}}^{T} \boldsymbol{v}_{j}=\delta_{i, j}
$$

where $\delta_{i, j}=1$ if $i=j$ and 0 otherwise. Then, the EVD of $\boldsymbol{S}$ is $\boldsymbol{S}=\sum_{k=2: p} \lambda_{k}\left(\boldsymbol{Q}^{T} \boldsymbol{v}_{k}\right)\left(\boldsymbol{Q}^{T} \boldsymbol{v}_{k}\right)^{T}$.

In the next section, based on this space reduction, we propose a method to track efficiently the leading eigenvector of matrix $S$ when first the global Laplacian matrix is known and second, when observing one node at a time through a random walk process. The particular choice that we made for matrix transform $Q$ is based on algorithmic complexity considerations, which we will discussed at the end of the next section.

\section{Tracking the leading eigenvector}

1) Known Laplacian case: The leading eigenvector $\boldsymbol{Q}^{T} \boldsymbol{v}_{2}$ of matrix $\boldsymbol{S}$ can be computed iteratively with a gradient descent approach applied to the Rayleigh quotient function given by Equation (1), or equivalently to the minimum eigenvalue approximation $\boldsymbol{x}^{T} \boldsymbol{S} \boldsymbol{x}$ with $\boldsymbol{x}$ constrained to be in the unit length vector manifold. Up to second order terms both approaches are equivalent and yield to the following update for $\boldsymbol{x}$ :

$$
\boldsymbol{x}_{k+1}=\boldsymbol{x}_{k}-\epsilon_{k}\left(\boldsymbol{S}-r_{\boldsymbol{S}}\left(\boldsymbol{x}_{k}\right)\right) \boldsymbol{x}_{k}, \boldsymbol{x}_{k+1}=\frac{\boldsymbol{x}_{k+1}}{\left\|\boldsymbol{x}_{k+1}\right\|},
$$

where $\epsilon_{k}$ is a decreasing step-size, such that $\sum_{k} \epsilon_{k}=+\infty$ and $\sum_{k} \epsilon_{k}^{2}<+\infty$ (typically, $\epsilon_{k}=\frac{1}{k+1}$ ). This latter method assumes complete knowledge of matrix $\boldsymbol{L}$ which implies the computation of matrix $\boldsymbol{S}$ following proposition 1. When the network is large or for particular reasons, it may not be possible to have such information. In the next section, we propose an iterative method when we have a partial observation of the matrix based on a random walk exploration of the network.

2) Random walk case: At each instant $k$, the random walk $X(k)$ is located at vertex $i_{k}$ and the neighboring nodes $\mathcal{N}\left(i_{k}\right)$ are observable. At instants $k$, line $i_{k}$ of $\boldsymbol{L}$ is observed and denoted by $l_{i_{k}}$. Then matrix $\boldsymbol{L}$ is replaced by the following instantaneous matrix $\boldsymbol{L}_{k}=\left(\mathbf{0}\left|\boldsymbol{l}_{i_{k}}\right| \mathbf{0}\right)^{T}$, where only line $i_{k}$ of $\boldsymbol{L}_{k}$ is nonzero. Then, the instantaneous version of matrix $\boldsymbol{S}$ is $\boldsymbol{S}_{k}=\boldsymbol{Q}^{T} \boldsymbol{L}_{k} \boldsymbol{Q}$. It should be noted that the probability to observe the nodes $i_{k}$ when moving on the graph according to a random walk is not uniform. Thus $\mathbb{E}\left[\boldsymbol{S}_{k}\right] \neq \boldsymbol{S}$ and this should be accounted to avoid asymptotically biased estimates. Indeed,

$$
\mathbb{E}\left[\boldsymbol{S}_{k}\right]=\mathbb{E}\left[\mathbb{E}\left[\boldsymbol{S}_{k} \mid i_{k}\right]\right]=\sum_{i=1: n} \pi_{i, k} \boldsymbol{S}_{k} \neq \frac{1}{n} \boldsymbol{S}
$$

where $\boldsymbol{\pi}_{k}=\left[\pi_{1, k}, \ldots, \pi_{n, k}\right]^{T}$ is the instantaneous distribution of the Markov process $X$. Unfortunately, as the topology is unknown, neither instantaneous nor asymptotic distributions of this Markov process are known. Then instantaneous estimate 
$\boldsymbol{p}_{i, k}$ of $\pi_{i, k}$ is updated at instant $k$ in our procedure as we explore the network by the random walk.

Let $\boldsymbol{p}_{0} \in \mathbb{R}_{+}^{n}$, with $\mathbb{I}^{T} \boldsymbol{p}_{0}=1, \boldsymbol{y}_{0} \in U^{n-1}$ and $\lambda_{0} \in \mathbb{R}_{+}$denote initial values. Then, at time $k+1(k \geq 0)$ the random walk $X$ visits node $i_{k+1}$ and updates are defined as follows:

$$
\begin{aligned}
\boldsymbol{p}_{k+1} & =\boldsymbol{p}_{k}+\epsilon_{p, k}\left(\boldsymbol{e}_{i_{k+1}}-\boldsymbol{p}_{k}\right) \\
\boldsymbol{x}_{k+1} & =\boldsymbol{Q} \boldsymbol{y}_{k}, \\
\boldsymbol{y}_{k+1} & =\boldsymbol{y}_{k}-\epsilon_{k}\left(\left[\boldsymbol{Q}^{T}\right]_{i_{k+1}} \frac{\boldsymbol{l}_{i_{k+1}} \boldsymbol{x}_{k+1}}{n p_{i_{k+1}, k+1}}-\lambda_{k} \boldsymbol{y}_{k}\right), \\
\boldsymbol{y}_{k+1} & =\left\|\boldsymbol{y}_{k+1}\right\|^{-1} \boldsymbol{y}_{k+1}, \\
\lambda_{k+1} & =\lambda_{k}+\epsilon_{\lambda, k}\left(\left[\boldsymbol{x}_{k+1}\right]_{i_{k+1}} \frac{\boldsymbol{l}_{i_{k+1}} \boldsymbol{x}_{k+1}}{n p_{i_{k+1}, k+1}}-\lambda_{k}\right),
\end{aligned}
$$

where the step-size sequences $\left\{\epsilon_{p, k}\right\},\left\{\epsilon_{k}\right\}$ and $\left\{\epsilon_{\lambda, k}\right\}$ should be such that

$$
\sum_{k} \epsilon_{p, k}=\sum_{k} \epsilon_{k}=\sum_{k} \epsilon_{\lambda, k}=+\infty, \sum_{k} \epsilon_{p, k}^{2}+\epsilon_{k}^{2}+\epsilon_{\lambda, k}^{2}<+\infty,
$$

and satisfy also the condition: $\frac{\epsilon_{k}}{\epsilon_{p, k}} \rightarrow 0, \frac{\epsilon_{k}}{\epsilon_{\lambda, k}} \rightarrow 0$. These conditions are satisfied considering $\epsilon_{k}=\frac{1}{1+k \log (1+k)}$ and $\epsilon_{\lambda, k}=\epsilon_{p, k}=\frac{1}{k+1}$. The latest conditions on the step-size sequences will ensure that the evolution of the sequence $y_{k}$ is much slower that the evolution of the sequences $p_{k}$ and $\lambda_{k}$. Therefore it implies that: (1) $\mathbf{p}_{k}$ have converged to the stationary distribution of the Markov process $X$; (2) the iterative procedure can be viewed as a noisy discretization of the following limiting system of a two-time scale ordinary differential equations (see ch.6 in [25]):

$$
\dot{\boldsymbol{y}}=-\left(\boldsymbol{Q}^{T} \boldsymbol{L} \boldsymbol{Q} \boldsymbol{y}-\lambda \boldsymbol{y}\right) \quad \text { and } \quad \lambda=\boldsymbol{y}^{T} \boldsymbol{Q}^{T} \boldsymbol{L} \boldsymbol{Q} \boldsymbol{y}
$$

This O.D.E. is equivalent to the following Rayleight Quotient Flow (2):

$$
\dot{\boldsymbol{y}}=-\left(\boldsymbol{Q}^{T} \boldsymbol{L} \boldsymbol{Q}-\boldsymbol{y}^{T} \boldsymbol{Q}^{T} \boldsymbol{L} \boldsymbol{Q} \boldsymbol{y}\right) \boldsymbol{y}=-\left(\boldsymbol{S}-\boldsymbol{y}^{T} \boldsymbol{S} \boldsymbol{y}\right) \boldsymbol{y}
$$

This fact will ensure the convergence of our scheme using the theory of stochastic approximation on manifold [11] to the leading eigenvector $\boldsymbol{w}_{2}$ corresponding to the highest eigenvalue $\lambda_{2}$ of the matrix $\boldsymbol{S}$. Then, the Fiedler vector $\boldsymbol{v}_{2}$ is obtained as $\boldsymbol{v}_{2}=\boldsymbol{Q}^{T} \boldsymbol{w}_{2}$.

Remark about the complexity per iteration: Simplifications in expressions involving $\boldsymbol{S}_{k}=\boldsymbol{Q}^{T} \boldsymbol{L}_{i_{k}} \boldsymbol{Q}$ where $\boldsymbol{L}_{i_{k}}$ only has one nonzero line and lead to linear complexity. In addition, although computations of the form $Q \boldsymbol{y}$ are involved in the algorithm, this does not yields high numerical burden due to the particular structure of matrix $Q$. Indeed, $Q$ only has two distinct nonzero terms in each of its columns. Thus computation of $\boldsymbol{Q} \boldsymbol{y}$ only involves about $2 n$ product terms, hence linear calculation complexity.

\section{NUMERICAL ILLUSTRATIONS}

In our simulations, for known $\boldsymbol{L}$ of size $n=50$ we choose $\epsilon_{k}=10^{2} /(k \log (k+1)+1)$. The ratio of the smallest eigenvalues of $\boldsymbol{S}$ is about 0.93 and after 300 iterations norm error is about $10^{-11}$.

For the same $\boldsymbol{L}$, when it is unknown and we use a random walk, stepsizes are set to $\epsilon_{k}=10^{2} /(k \log (k+1)+1)$, $\epsilon_{p, k}=1 /(k+1)$ and $\epsilon_{\lambda, k}=10^{-2} /(k+1)$. After $10^{6}$ iterations the MSE $\mathbb{E}\left[\left\|\boldsymbol{v}_{2}-\boldsymbol{x}\right\|^{2}\right]$, computed on the last half of estimates, is about $10^{-2}$.

One reason for the increase of the number of iterations compared to the case where $\boldsymbol{L}$ is known at each instant is because only a fraction $1 / n$ of the matrix $\boldsymbol{L}$ is observed at each time in the case of a random walk on the graph. From earlier discussion it also appears that computational complexity of loops is also roughly reduced by a factor proportional to $n$ in the case of line observations.

\section{Conclusions And Perspectives}

In this paper, we have described an iterative scheme that converges to the Fiedler value and the Fiedler vector of a network using information gathered by a random walk. The follow-up of this work will address (i) the characterization of the exact rate of convergence of this algorithm, (ii) the extension of this algorithm to a set-up where we suppose that the number of nodes is not known in advance and (iii) the extension of this work to a set-up where the network is evolving over time. 

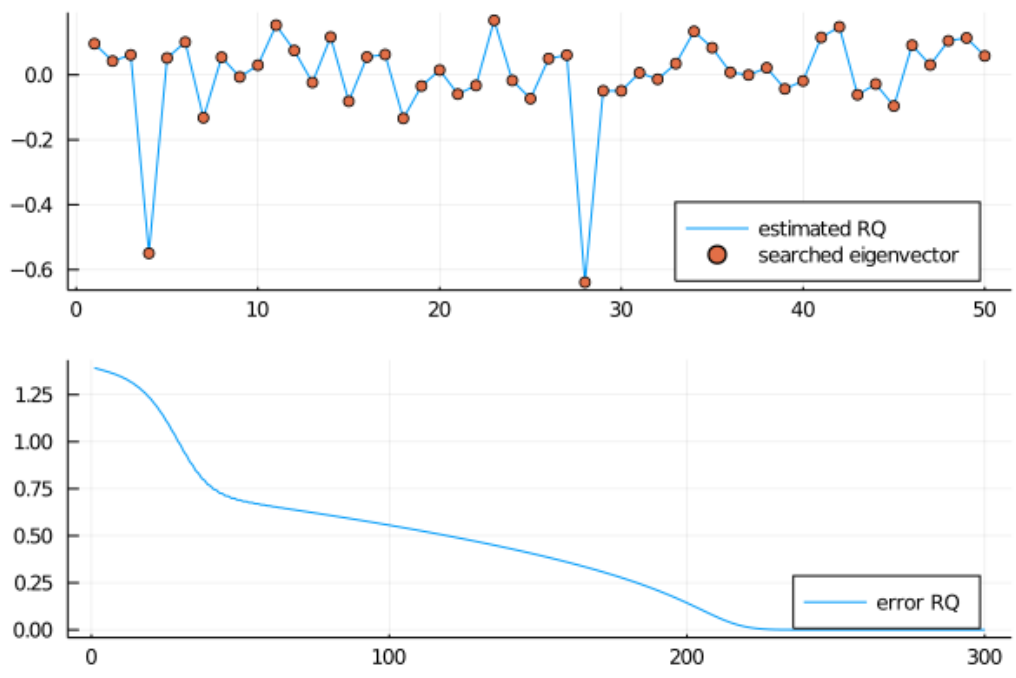

Fig. 1. Tracking with known $\boldsymbol{L}$.
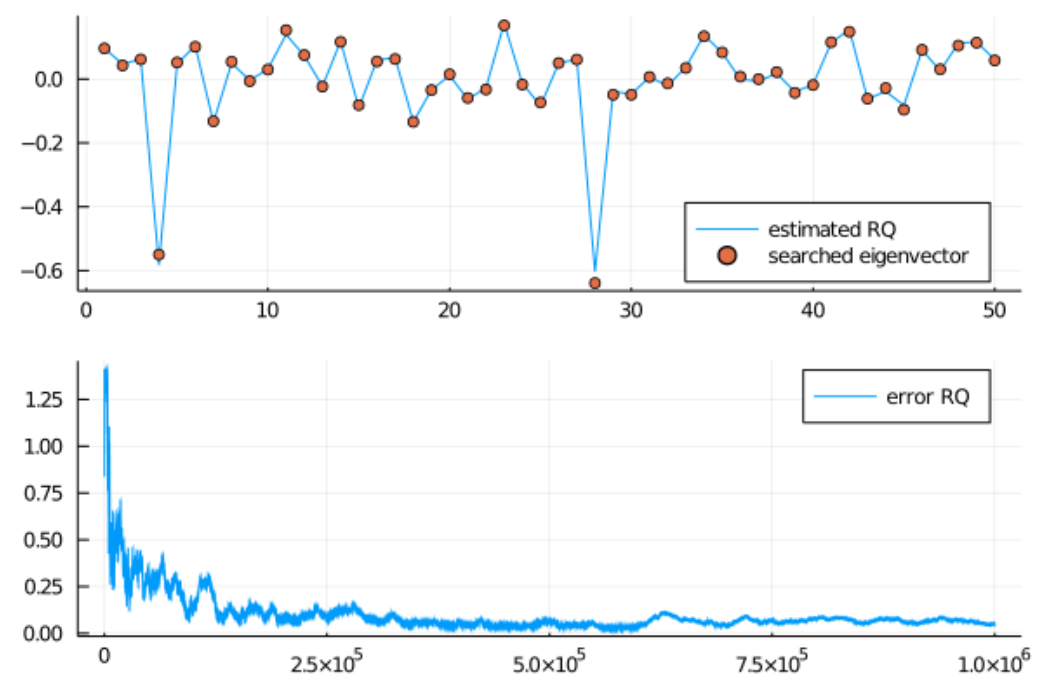

Fig. 2. Tracking with random walk exploration.

\section{REFERENCES}

[1] Christos Gkantsidis, Gagan Goel, Milena Mihail, and Amin Saberi, "Towards topology aware networks," in IEEE INFOCOM 2007-26th IEEE International Conference on Computer Communications. IEEE, 2007, pp. 2591-2595.

[2] Paolo Di Lorenzo and Sergio Barbarossa, "Distributed estimation and control of algebraic connectivity over random graphs," IEEE Transactions on Signal Processing, vol. 62, no. 21, pp. 5615-5628, 2014.

[3] Sergio Barbarossa, Stefania Sardellitti, and Paolo Di Lorenzo, "Distributed detection and estimation in wireless sensor networks," vol. 2, pp. 329-408, 2014.

[4] Paolo Di Lorenzo, Sergio Barbarossa, and Ali H Sayed, "Bio-inspired decentralized radio access based on swarming mechanisms over adaptive networks," IEEE Transactions on Signal Processing, vol. 61, no. 12, pp. 3183-3197, 2013.

[5] Fan RK Chung and Fan Chung Graham, Spectral graph theory, Number 92. American Mathematical Soc., 1997.

[6] Ulrike Von Luxburg, "A tutorial on spectral clustering," Statistics and computing, vol. 17, no. 4, pp. 395-416, 2007.

[7] Alexander Bertrand and Marc Moonen, "Seeing the bigger picture: How nodes can learn their place within a complex ad hoc network topology," IEEE Signal Processing Magazine, vol. 30, no. 3, pp. 71-82, 2013

[8] Arpita Ghosh and Stephen Boyd, "Growing well-connected graphs," in Proceedings of the 45th IEEE Conference on Decision and Control. IEEE, 2006, pp. 6605-6611.

[9] Miroslav Fiedler, "Algebraic connectivity of graphs," Czechoslovak mathematical journal, vol. 23, no. 2, pp. 298-305, 1973.

[10] Atish Das Sarma, Danupon Nanongkai, Gopal Pandurangan, and Prasad Tetali, "Distributed random walks," Journal of the ACM (JACM), vol. 60, no. 1, pp. 1-31, 2013

[11] Suhail M Shah, "Stochastic approximation on riemannian manifolds," Applied Mathematics \& Optimization, pp. 1-29, 2019.

[12] Peter Arbenz, Daniel Kressner, and DME Zürich, "Lecture notes on solving large scale eigenvalue problems," D-MATH, EHT Zurich, vol. 2 , 2012. 
[13] Dan Garber, Elad Hazan, and Tengyu Ma, "Online learning of eigenvectors.," in ICML, 2015, pp. 560-568.

[14] Ioannis Mitliagkas, Constantine Caramanis, and Prateek Jain, "Memory limited, streaming pca," in Advances in neural information processing systems, 2013, pp. 2886-2894.

[15] Prateek Jain, Chi Jin, Sham M Kakade, Praneeth Netrapalli, and Aaron Sidford, "Streaming pca: Matching matrix bernstein and near-optimal finite sample guarantees for oja's algorithm," in Conference on learning theory, 2016, pp. 1147-1164.

[16] Alexander Bertrand and Marc Moonen, "Distributed computation of the fiedler vector with application to topology inference in ad hoc networks," Signal Processing, vol. 93, no. 5, pp. 1106-1117, 2013.

[17] Konstantin Avrachenkov, Philippe Jacquet, and Jithin K Sreedharan, "Distributed spectral decomposition in networks by complex diffusion and quantum random walk," in IEEE INFOCOM 2016-The 35th Annual IEEE International Conference on Computer Communications. IEEE, 2016, pp. 1-9.

[18] Vivek S Borkar, Rahul Makhijani, and Rajesh Sundaresan, "Asynchronous gossip for averaging and spectral ranking," IEEE Journal of Selected Topics in Signal Processing, vol. 8, no. 4, pp. 703-716, 2014.

[19] V Borkar and Sean P Meyn, "Oja's algorithm for graph clustering, markov spectral decomposition, and risk sensitive control," Automatica, vol. 48, no. 10, pp. 2512-2519, 2012

[20] Vishwaraj Doshi and Do Young Eun, "Fiedler vector approximation via interacting random walks," Proceedings of the ACM on Measurement and Analysis of Computing Systems, vol. 4, no. 1, pp. 1-28, 2020.

[21] Małgorzata Lucińska and Sławomir T Wierzchoń, "Graph clustering using early-stopped random walks," in IFIP International Conference on Computer Information Systems and Industrial Management. Springer, 2016, pp. 416-428.

[22] Piet Van Mieghem, Graph spectra for complex networks, Cambridge University Press, 2010.

[23] G. H. Golub and C. F. Van Loan, Matrix Computations, Johns Hopkins Press, 1989.

[24] Uwe Helmke and John B Moore, Optimization and dynamical systems, Springer Science \& Business Media, 2012.

[25] Vivek S Borkar, Stochastic approximation: a dynamical systems viewpoint, vol. 48, Springer, 2009. 